\title{
Some non-selfadjoint problems on the circle *
}

José A. Lubary, Dept. Matemàtica Aplicada II, Universitat Politècnica de Catalunya, 08028-Barcelona

Joan de Solà-Morales, Dept. Matemàtica Aplicada I, Universitat Politècnica de Catalunya, 08028-Barcelona

\begin{abstract}
The non-selfadjoint nature of some second order differential equations with periodic boundary conditions is exhibited by describing properties of its spectrum, such as geometric and algebraic multiplicities and existence of nonreal eigenvalues.
\end{abstract}

Keywords: non-selfadjoint operators, periodic boundary conditions, nonreal eigenvalues, multiplicities.

AMS subject classification: Primary 34B, Secondary 47.

\section{Introduction}

Consider the eigenvalue problem

$$
a(x) u^{\prime \prime}(x)+b(x) u^{\prime}(x)+c(x) u(x)+\lambda u(x)=0, \quad x_{1} \leq x \leq x_{2} ;
$$

where $a, b, c$ are real functions on $\left[x_{1}, x_{2}\right]$ and $a(x) \geq \epsilon>0$ for some $\epsilon$, with the following conditions:

$$
u\left(x_{1}\right)=u\left(x_{2}\right) ; \quad \alpha_{1} u^{\prime}\left(x_{1}\right)+\beta u\left(x_{1}\right)=\alpha_{2} u^{\prime}\left(x_{2}\right),
$$

where $\alpha_{1}, \alpha_{2}$ and $\beta$ are real numbers and $\alpha_{1}, \alpha_{2}>0$ (the case $\alpha_{1}=\alpha_{2}, \beta=0$ corresponds to the periodic problem). Without loss of generality we can suppose $\left[x_{1}, x_{2}\right]=[0,1]$.

${ }^{*}$ Partially supported by the UPC grant 642.2 and the DGICYT project PB95-0629-C02-02, Spain 
It is known that the equation (1.1) admits a formally selfadjoint form

$$
\left(p(x) u^{\prime}(x)\right)^{\prime}+q(x) u(x)+\lambda \gamma(x) u(x)=0,
$$

where $p(x)>0$ and $\gamma(x)>0$ for $0 \leq x \leq 1$, and, if

$$
\int_{0}^{1} \frac{b(x)}{a(x)} d x=\ln \frac{\alpha_{2}}{\alpha_{1}}
$$

then the operator $T$ of $L^{2}(0,1)$ defined by $T u=\frac{1}{\gamma}\left(\left(p u^{\prime}\right)^{\prime}+q u\right)$ with

$$
D(T)=\left\{u \in H^{2}(0,1) \mid u(0)=u(1) ; \alpha_{1} u^{\prime}(0)+\beta u(0)=\alpha_{2} u^{\prime}(1)\right\}
$$

is selfadjoint for the metric of $L^{2}(0,1)$ defined by $(u, v)=\int_{0}^{1} \gamma u \bar{v} d x$ and has an infinite number of real eigenvalues with geometric multiplicities equal to 1 or 2, and for every eigenvalue the algebraic and geometric multiplicities coincide.

We are going to investigate the case in which the condition (1.3) fails to hold. Assume

$$
2 k:=\int_{0}^{1} \frac{b(x)}{a(x)} d x-\ln \frac{\alpha_{2}}{\alpha_{1}} \neq 0 ;
$$

it is easy to see that, in this case, $T$ is not symmetric (and consequently it is not selfadjoint) for the metric defined above.

The purpose of this paper is to study for this problem the typical phenomena that are associated to non-selfadjointness: existence of (nonreal) complex eigenvalues and existence of eigenvalues with algebraic multiplicity larger than the geometric multiplicity. Our motivation comes from the belief that the study of linear second-order differential operators on graphs (see [2]) has to give some light for an understanding of the properties of the second-order elliptic operators in open domains of $\mathbb{R}^{n}$ for $n \geq 2$, as in [3] and [8], where self-adjointness is not generally to be expected (unlike for the interval, for $n=1$ ). So, we see our results as a contribution to the view of graphs as one-dimensional objects that exhibit some of the features of the more-dimensional domains, and among the nontrivial graphs, the circle is the simplest case. In this sense our paper was motivated by a previous paper by the first author [11] for general graphs, where only the geometric multiplicity was studied.

In sections 2 and 5 we make the hypothesis that the coefficient functions $a(x)$, $b(x)$ and $c(x)$ that appear in (1.1) are in $L^{1}(0,1)$, and in sections 3 and 4 that they are functions of bounded variation. These are quite general assumptions and are motivated by the fact that discontinuous coefficients appear very naturally in applications as a consequence of changes of materials along the circle. Another reason is the natural discontinuity in the coefficient functions that will appear below when we will extend them to all $x \in \mathbb{R}$ by periodicity. Because of these assumptions, the equation (1.1) has to be understood in a weak sense: the solution 
must be a $\mathcal{C}^{1}$ function of $x$ whose second derivative in the generalized sense satisfies the equation. For these kind of solutions one can see that one has the usual results of existence and uniqueness for the initial value problem, but details will not be presented here. It is also clear that any change in the coefficient functions on a set of zero measure does not change the solutions at all.

\section{Normalized form, and summary of results}

In order to work with the simplest possible equation, we are going to define a kind of normalized form. First of all, by performing a change of variables in (1.1)-(1.2) of the type

$$
x=f(s), v(s)=u(f(s)) \Psi(s),
$$

with $f$ and $\Psi$ in $\mathcal{C}^{\infty}[0,1]$ such that

$$
\begin{gathered}
f(0)=0, f(1)=1, f^{\prime}(s)>0(0 \leq s \leq 1), \alpha_{2} f^{\prime}(0)=\alpha_{1} f^{\prime}(1), \\
\Psi(s) \neq 0(0 \leq s \leq 1), \Psi(0)=\Psi(1), \text { and } \Psi^{\prime}(0)-\Psi^{\prime}(1)=\frac{\beta}{\alpha_{2}} \Psi(1) f^{\prime}(1),
\end{gathered}
$$

the problem becomes a new equation

$$
\tilde{a}(s) v^{\prime \prime}(s)+\tilde{b}(s) v^{\prime}(s)+\tilde{c}(s) v(s)+\lambda v(s)=0,
$$

but now with periodic boundary conditions. It is easy to see that

$$
\int_{0}^{1} \frac{\tilde{b}(s)}{\tilde{a}(s)} d s=\int_{0}^{1} \frac{b(x)}{a(x)} d x-\ln \frac{\alpha_{2}}{\alpha_{1}}=2 k .
$$

Performing now a new change in the independent variable of the form $p(s) d t=d s$ with a suitable positive and periodic function $p(s)$ the problem (1.1)-(1.2) finally becomes

$$
\begin{gathered}
v^{\prime \prime}(t)+2 k v^{\prime}(t)+q(t) v(t)+\lambda r(t) v(t)=0 \\
v(0)=v(1) ; v^{\prime}(0)=v^{\prime}(1)
\end{gathered}
$$

with $r(t)>0$ for $0 \leq t \leq 1$.

It is known (see [10]) that if $r(t)$ is smooth and periodic, one can find a new change of variables such that the problem (2.1)-(2.2) keeps the same form except for changes in the function $q$ and that the new funcion $r$ becomes the function constantly equal to 1 . This seems not to be possible for a general $r(t)$ (see Theorem 3 and Remark 1 below), but some more information on this type of changes of variables can be found in Remark 2 .

For $k=0$, the problem (1.1)-(1.2) admits an infinite number of real eigenvalues, as we can see in [5], an the operator defined in the previous section is selfadjoint for the metric also defined there. 
Putting now $w=e^{k t} v$, we obtain what we call the normalized form

$$
\begin{aligned}
& w^{\prime \prime}(t)+h(t) w(t)+\lambda r(t) w(t)=0 ; \\
& w(1)=e^{k} w(0) ; w^{\prime}(1)=e^{k} w^{\prime}(0) .
\end{aligned}
$$

Now we explain the contents of the rest of the paper. In section 3 we prove that our problem admits an infinite number of eigenvalues. In section 4 one sees that for the problem in the normalized form, for large values of $k$ there is only one real eigenvalue. One also sees that for $r(t)$ smooth and periodic and arbitrary $k \neq 0$ there are only finitely many real eigenvalues, but we show by an example that when $r(t)$ is not so regular this may not be the case. Finally, section 5 is devoted to the problem of the algebraic and geometric multiplicities. The results obtained in this last section coincide with those that can be deduced from a more general problem that is studied in [1], for the case of real analytic and periodic coefficients, or in [4], for the case $r(t) \equiv 1$ and $h(t)$ in $L^{\infty}(0,1)$. In comparison with these two works, in the present paper we do not obtain the oscillation properties of the eigenfunctions, but we present much more direct proofs of the multiplicity results.

\section{Existence of an infinite number of eigenvalues}

Consider the problem (with $k \neq 0$ and $r(t)>0$ )

$$
\begin{gathered}
u^{\prime \prime}(t)+h(t) u(t)+\lambda r(t) u(t)=0,0 \leq t \leq 1 \\
u(1)=e^{k} u(0) ; u^{\prime}(1)=e^{k} u^{\prime}(0) .
\end{gathered}
$$

We call $B V(0,1)$ the class of functions of bounded variation in $(0,1)$ (see [7]).

Theorem 1 Suppose $h, r \in B V(0,1)$. There exists an infinite number of eigenvalues for the problem (3.1)-(3.2).

Proof Suppose first that $h$ and $r$ are in $\mathcal{C}^{\infty}[0,1]$. Let $\phi$ and $\psi$ be the solutions (obviously depending on $\lambda$ ) of (3.1) such that $\phi(0)=1, \phi^{\prime}(0)=0, \psi(0)=0, \psi^{\prime}(0)=1$. Any other solution of (3.1) can be written as $u(t)=C_{1} \phi(t)+C_{2} \psi(t)$. By imposing the conditions (3.2) we obtain for $C_{1}$ and $C_{2}$ the algebraic linear system

$$
C_{1} \phi(1)+C_{2} \psi(1)=e^{k} C_{1} ; \quad C_{1} \phi^{\prime}(1)+C_{2} \psi^{\prime}(1)=e^{k} C_{2} .
$$

By using that $\phi(t) \psi^{\prime}(t)-\phi^{\prime}(t) \psi(t)=1$ we can see that this system has non trivial solutions if and only if

$$
\phi(1, \lambda)+\psi^{\prime}(1, \lambda)=2 \cosh k .
$$

Let $f$ be the function defined by $f(\lambda)=\phi(1, \lambda)+\psi^{\prime}(1, \lambda) ; f$ is an entire function of $\lambda$, as we can see in [5]. 
For our purposes we restrict ourselves to values of $\lambda$ such that

$$
|\lambda|>\frac{1+|h|_{\max }}{r_{\min }}
$$

(this implies, in particular, that $h(t)+r(t) \lambda \neq 0$ for all $t$ ). The auxiliar equation

$$
v^{\prime \prime}(t)-\frac{g^{\prime}(t)}{2 g(t)} v^{\prime}(t)+g(t) v(t)=0,
$$

with $g(t)=h(t)+r(t) \lambda$, admits the independent solutions $e^{i \omega(t)}$ and $e^{-i \omega(t)}$, with $\omega$ such that $\omega^{\prime}(t)=\sqrt{g(t)}$ and $\omega(0)=0$. We write a solution of (3.1) in the form $u(t)=a(t) e^{i \omega(t)}+b(t) e^{-i \omega(t)}$, where $a(t)$ and $b(t)$ are complex-valued and $a^{\prime}(t) e^{i \omega(t)}+b^{\prime}(t) e^{-i \omega(t)}=0$. By substituting in (3.1) we obtain easily

$$
a^{\prime}(t) e^{i \omega(t)}-b^{\prime}(t) e^{-i \omega(t)}=-\frac{g^{\prime}(t)}{2 g(t)}\left(a(t) e^{i \omega(t)}-b(t) e^{-i \omega(t)}\right) .
$$

We call now $R(t)=a(t) e^{i \omega(t)}+b(t) e^{-i \omega(t)}$ and $S(t)=a(t) e^{i \omega(t)}-b(t) e^{-i \omega(t)}$; we obtain for $R$ and $S$ :

$$
R^{\prime}(t)=i \omega^{\prime}(t) S(t) ; \quad S^{\prime}(t)=-\frac{g^{\prime}(t)}{2 g(t)} S(t)+i \omega^{\prime}(t) R(t) .
$$

An easy calculation shows that

$\frac{d}{d t}\left(|R(t)|^{2}+|S(t)|^{2}\right)=-R e\left(\frac{g^{\prime}(t)}{g(t)}\right)|S(t)|^{2}-2 \operatorname{Im}\left(\omega^{\prime}(t)\right)(\overline{R(t)} S(t)+R(t) \overline{S(t)}) ;$

therefore

$$
\begin{aligned}
\frac{d}{d t}\left(|R(t)|^{2}+|S(t)|^{2}\right) & \leq\left|\frac{d}{d t}\left(|R(t)|^{2}+|S(t)|^{2}\right)\right| \\
& \leq\left(\left|\frac{g^{\prime}(t)}{g(t)}\right|+2|\sqrt{g(t)}|\right)\left(|R(t)|^{2}+|S(t)|^{2}\right) .
\end{aligned}
$$

Then, we deduce

$$
|R(1)|^{2}+|S(1)|^{2} \leq\left(|R(0)|^{2}+|S(0)|^{2}\right) e^{Q(\lambda)},
$$

where

$$
Q(\lambda)=\int_{0}^{1}\left|\frac{g^{\prime}(t)}{g(t)}\right| d t+2 \int_{0}^{1}|\sqrt{g(t)}| d t .
$$

If we consider now the cases $u=\phi$ and $u=\psi$, we find

$$
|\phi(1)| \leq e^{\frac{1}{2} Q(\lambda)} ; \quad\left|\psi^{\prime}(1)\right| \leq\left|\sqrt{\frac{g(1)}{g(0)}}\right| e^{\frac{1}{2} Q(\lambda)},
$$


and then

$$
|f(\lambda)| \leq\left(1+\left|\sqrt{\frac{g(1)}{g(0)}}\right|\right) e^{\frac{1}{2} Q(\lambda)}
$$

It is easy to see that, for $|\lambda|>\frac{1+|h|_{\max }}{r_{\min }}$, the following inequalities hold:

$$
\begin{gathered}
\mid \sqrt{\frac{g(1)}{g(0)} \mid} \leq \sqrt{|h|_{\text {max }}+\frac{r_{\text {max }}}{r_{\text {min }}}\left(1+|h|_{\text {max }}\right)}, \\
\int_{0}^{1}\left|\frac{g^{\prime}(t)}{g(t)}\right| d t \leq V(h)+\frac{1+|h|_{\text {max }}}{r_{\text {min }}} V(r), \\
\int_{0}^{1}|\sqrt{g(t)}| d t \leq \sqrt{|\lambda|} \sqrt{r_{\text {max }}+\frac{|h|_{\text {max }}}{1+|h|_{\text {max }}} r_{\text {min }}},
\end{gathered}
$$

where $V(h)$ and $V(r)$ are, respectively, the total variations of the funcions $h$ and $r$ (see [7]). So, it is clear that there exist positive real numbers $M$ and $N$ such that

$$
|f(\lambda)| \leq M e^{N \sqrt{|\lambda|}}
$$

The following lemma allows us to extend the result (3.7) to the general case of $h$ and $r$ of bounded variation. The proof, that is based on ideas from [7], will be omitted here, but will appear in [12].

Lemma 1 Let $F$ be a function of bounded variation on $[0,1]$ and suppose $F(t) \geq$ $\epsilon>0$ for all $t \in[0,1]$. Then, there exists a sequence of functions $\left\{F_{n}\right\}$ in $\mathcal{C}^{\infty}[0,1]$ and a positive number $C_{F}$ such that

$$
\begin{gathered}
\lim _{n \rightarrow \infty} \int_{0}^{1}\left|F_{n}(t)-F(t)\right| d t=0, \quad V\left(F_{n}\right) \leq C_{F} \text { for all } n \\
\quad \text { and } \frac{1}{C_{F}} \leq F_{n}(t) \leq C_{F} \text { for all } n \text { and } t
\end{gathered}
$$

(Obviously we can apply this to a non-positive function by adding an appropriate constant).

Let $\left\{h_{n}\right\}$ and $\left\{r_{n}\right\}$ be the sequences obtained by applying Lemma 1 to $h$ and $r$ of bounded variation. We consider the equations

$$
u_{n}^{\prime \prime}(t)+h_{n}(t) u_{n}(t)+\lambda r_{n}(t) u_{n}(t)=0,0 \leq t \leq 1
$$

with the same initial conditions as for $u$. By applying the Gronwall inequality to the difference $u_{n}-u$ it can be shown that $\phi_{n}(1) \rightarrow \phi(1)$ and $\psi_{n}^{\prime}(1) \rightarrow \psi^{\prime}(1)$, so we 
have in the limit the same left hand for the inequality (3.7). Moreover, the lemma also allows us to keep bounded in the limit the right hands of the inequalities (3.6), and so (3.7) is also true when $h$ and $r$ are of bounded variation.

Now we are going to study the behavior of $|f(\lambda)|$ for $\lambda$ real and going to $-\infty$. Let $K$ be a real number and suppose that we choose $\lambda$ (real) such that

$$
-\delta K^{2}<h(t)+r(t) \lambda<-K^{2} \text { for all } t
$$

(this is possible if we take $\delta=\sup (r) / \inf (r)$ ). The $\phi$ and $\psi$ for the equation

$$
u^{\prime \prime}(t)-K^{2} u(t)=0
$$

are $\phi_{K}(t)=\cosh (K t)$ and $\psi_{K}(t)=(1 / K) \sinh (K t)$.

By multiplying the equation $\phi^{\prime \prime}(t)+(h(t)+r(t) \lambda) \phi(t)=0$ by $\phi_{K}(t)$ and the equation (3.8) by $\phi(t)$, substracting and integrating by parts we obtain

$$
\phi^{\prime}(t) \phi_{K}(t)-\phi_{K}^{\prime}(t) \phi(t)=-\int_{0}^{t}\left(h(s)+r(s) \lambda+K^{2}\right) \phi(t) \phi_{K}(t) d s .
$$

First, it is clear that $\phi(t)>0$ for all $t$; otherwise, if $t_{0}$ is the first zero of $\phi$ we have $\phi\left(t_{0}\right)=0$ and $\phi^{\prime}\left(t_{0}\right)<0$, but if we put $t_{0}$ in the place of $t$ in (3.9) we obtain $\phi^{\prime}\left(t_{0}\right)>0$. So, it is clear that $\phi^{\prime}(t) \phi_{K}(t)-\phi_{K}^{\prime}(t) \phi(t)>0$ for all $t$, and then $\left(\phi / \phi_{K}\right)^{\prime}(t)>0$ for all $t$. We deduce easily $\phi(1)>\phi_{K}(1)=\cosh K$. In a similar way we can deduce $\psi^{\prime}(1)>\psi_{K}^{\prime}(1)=\cosh K$, and so

$$
f(\lambda)>2 \cosh K>e^{K}>\exp \sqrt{-\frac{1}{\delta}(\inf (h)+\lambda \sup (r))},
$$

and then it is clear that there exists a positive real number $m$ such that

$$
\lim _{\lambda \rightarrow-\infty}|f(\lambda)| e^{-m \sqrt{|\lambda|}}=\infty .
$$

An entire function satisfying (3.7) and (3.10) is said to have order of growth $1 / 2$. A well known theorem on complex variable functions says that an entire function with non integer order of growth has an infinite number of zeroes (see [6]). Since $f(\lambda)-2 \cosh k$ satisfies these hypotheses, this proves the theorem.

\section{Existence of nonreal eigenvalues}

Theorem 2 Suppose $h, r \in B V(0,1)$. There exists $k_{0}>0$ such that, if $|k|>k_{0}$, then there is only one real eigenvalue for the problem (3.1)-(3.2).

Proof If we substitute $\lambda$ by $C \lambda$ or $C+\lambda$ with $C$ an arbitrary constant, an equivalent problem is obtained. So we can assume $0<\inf (r) \leq \sup (r)=1$ and $\inf (h)=1+\frac{\pi^{2}}{4}$. 
Let $\mu_{0}$ be the first eigenvalue of (3.1) with $u(0)=0, u^{\prime}(1)=0$ and $\nu_{0}$ the first one with $u^{\prime}(0)=0, u(1)=0$. We call $\lambda_{0}=\min \left(\mu_{0}, \nu_{0}\right)$. It is easy to see, by using the Rayleigh quotient, that $\lambda_{0} \leq-1$.

Let us analyze the function $f(\lambda)=\phi(1, \lambda)+\psi^{\prime}(1, \lambda)$ for real values of $\lambda$.

(i) Case $\lambda>\left|\lambda_{0}\right|$.

As before, we consider first $h$ and $r$ in $C^{\infty}[0,1]$ and extend later the result to the case of bounded variation.

For briefness we call $g(t)=h(t)+r(t) \lambda$. The auxiliar equation (3.5) admits now the solutions $v_{1}=\sin \omega(t)$ and $v_{2}=\cos \omega(t)$, with $\omega(t)$ such that $\omega^{\prime}(t)=\sqrt{g(t)}$ and $\omega(0)=0$. Let us write a solution of $(3.1)$ in the form $u(t)=a(t) v_{1}(t)+b(t) v_{2}(t)$. We can easily see that we can write for $a, b$

$$
a^{\prime} v_{1}+b^{\prime} v_{2}=0 ; \quad a^{\prime} v_{1}^{\prime}+b^{\prime} v_{2}^{\prime}=-\frac{g^{\prime}}{2 g}\left(a v_{1}^{\prime}+b v_{2}^{\prime}\right) .
$$

Then,

$$
a a^{\prime}+b b^{\prime}=-\frac{g^{\prime}}{2 g}(a \cos \omega+b \sin \omega)^{2}
$$

therefore,

$$
\left(a(t)^{2}+b(t)^{2}\right)^{\prime} \leq\left|\left(a(t)^{2}+b(t)^{2}\right)^{\prime}\right| \leq\left|\frac{g^{\prime}(t)}{g(t)}\right|\left(a(t)^{2}+b(t)^{2}\right) ;
$$

and finally

$$
a(1)^{2}+b(1)^{2} \leq\left(a(0)^{2}+b(0)^{2}\right) \exp \left(\int_{0}^{1}\left|\frac{g^{\prime}(t)}{g(t)}\right| d t\right) .
$$

Since

$$
\begin{aligned}
\int_{0}^{1}\left|\frac{g^{\prime}(t)}{g(t)}\right| d t & \leq \frac{1}{g_{\min }} \int_{0}^{1}\left|g^{\prime}(t)\right| d t=\frac{1}{g_{\min }} V(g) \\
& \leq \frac{V(h)+V(r) \lambda}{h_{\min }+r_{\min } \lambda}
\end{aligned}
$$

it is clear that there exists a constant $C$ (independent of $\lambda$ ) such that $a(1)^{2}+b(1)^{2} \leq$ $C\left(a(0)^{2}+b(0)^{2}\right)$. Then, by applying this to $\phi$ and $\psi$ we deduce easily

$$
\left|\phi(1, \lambda)+\psi^{\prime}(1, \lambda)\right| \leq 2 \cosh k_{1} \text { for some } k_{1}>0 .
$$

Now we can extend this inequality to $h, r \in B V(0,1)$ by using Lemma 1.

(ii) Case $-\left|\lambda_{0}\right| \leq \lambda \leq\left|\lambda_{0}\right|$.

Every solution of (3.1) can be written, by means of the so called Prüfer transformation, as $u=\rho \sin \omega$ with $u^{\prime}=\rho \cos \omega$, where $\rho>0$ and $\omega$ are solutions of

$$
\rho^{\prime}=(1-(h+r \lambda)) \rho \sin \omega \cos \omega,
$$




$$
\omega^{\prime}=\cos ^{2} \omega+(h+r \lambda) \sin ^{2} \omega
$$

From (4.2) we obtain

$$
\rho(t) \leq \rho(0) e^{\delta t}
$$

where $\delta=1+\sup (h)+\left|\lambda_{0}\right|$. Applying this to $\phi$ and $\psi$ we easily obtain

$$
\left|\phi(1, \lambda)+\psi^{\prime}(1, \lambda)\right| \leq 2 e^{\delta}=2 \cosh k_{2} \text { for some } k_{2}>0 .
$$

(iii) Case $\lambda<\lambda_{0}$.

By comparison arguments as those used at the end of the proof of Theorem 1 it is easy to see that

$$
\lambda_{1}<\lambda_{2}<\lambda_{0} \Rightarrow \phi\left(1, \lambda_{1}\right)>\phi\left(1, \lambda_{2}\right)>0 \text { and } \psi^{\prime}\left(1, \lambda_{1}\right)>\psi^{\prime}\left(1, \lambda_{2}\right)>0 .
$$

In other words, the function $f(\lambda)=\phi(1, \lambda)+\psi^{\prime}(1, \lambda)$ is, for $\lambda<\lambda_{0}$, positive and strictly decreasing. Now, if we take $\lambda$ such that $h(t)+r(t) \lambda<-k^{2}$, by comparison with the solutions $\phi_{k}=\cosh (k t)$ and $\psi_{k}=\frac{1}{k} \sinh (k t)$ of the equation $u^{\prime \prime}-k^{2} u=0$ we deduce $\phi(1, \lambda)>\phi_{k}(1)=\cosh k$ and $\psi^{\prime}(1, \lambda)>\psi_{k}^{\prime}(1)=\cosh k$ and then

$$
\phi(1, \lambda)+\psi^{\prime}(1, \lambda)>2 \cosh k .
$$

Call $k_{0}=\max \left(k_{1}, k_{2}\right)$. It is clear now that, for $k>k_{0}$, the curve $y=f(\lambda)$ intersects only once the straight line $y=2 \cosh k$, and this proves the theorem.

Theorem 3 For $h$ in $B V(0,1)$ and $r$ in $\mathcal{C}^{2}[0,1]$ with $r(0)=r(1), r^{\prime}(0)=r^{\prime}(1)$, and $k \neq 0$, there exists only a finite number of real eigenvalues for the problem (3.1)-(3.2).

Proof The change of variables

$$
\tau=R^{-1 / 2} \int_{0}^{t} \sqrt{r(s)} d s \text { with } R=\left(\int_{0}^{1} \sqrt{r(t)} d t\right)^{2} ; w(\tau)=r(t)^{1 / 4} u(t),
$$

transforms the problem (3.1)-(3.2) in

$$
\begin{gathered}
\frac{d^{2} w(\tau)}{d \tau^{2}}+(q(\tau)+\tilde{\lambda}) w(\tau)=0, \\
w(1)=e^{k} w(0) ; w^{\prime}(1)=e^{k} w(0),
\end{gathered}
$$

with $\tilde{\lambda}=R \lambda$. The problem (4.5)-(4.6) can be considered as a bounded perturbation (see [9]) of

$$
\begin{gathered}
\frac{d^{2} w(\tau)}{d \tau^{2}}+\tilde{\lambda} w(\tau)=0, \\
w(1)=e^{k} w(0) ; w^{\prime}(1)=e^{k} w(0),
\end{gathered}
$$

and every eigenvalue of (4.5)-(4.6) stays in a circle with center in the corresponding eigenvalue of (4.7)-(4.8) and a fixed radius depending only on $\sup |q(\tau)|$. Since the eigenvalues of (4.7)-(4.8) are $\tilde{\lambda}_{n}=4 n^{2} \pi^{2} \pm 4 n \pi k i$, we deduce that the eigenvalues of (4.5)-(4.6) are not real for large $n$. 
Remark 1 We can replace $\mathcal{C}^{2}[0,1]$ in Theorem 3 by $\mathcal{W}^{2,1}(0,1)$, but we cannot relax too much this condition, as we can see in the following example. Suppose $h(t)=0$ for all $t$ and $r(t)=r_{1}$ for $0 \leq t \leq 1 / 2, r(t)=r_{2}$ for $1 / 2 \leq t \leq 1$, with $r_{1}, r_{2}$ positive constants, and $r_{1} \neq r_{2}$. An elementary calculation shows that, in this case,

$$
f(\lambda)=2 \cos \frac{\sqrt{\lambda r_{1}}+\sqrt{\lambda r_{2}}}{2}-\delta \sin \frac{\sqrt{\lambda r_{1}}}{2} \sin \frac{\sqrt{\lambda r_{2}}}{2},
$$

where $\delta=\sqrt{r_{1} / r_{2}}+\sqrt{r_{2} / r_{1}}-2>0$. By putting

$$
s=\frac{\sqrt{\lambda r_{1}}+\sqrt{\lambda r_{2}}}{2} \text { and } \omega=\frac{\left|\sqrt{r_{1}}-\sqrt{r_{2}}\right|}{\sqrt{r_{1}}+\sqrt{r_{2}}}
$$

we can write the right hand of (4.9) in the form

$$
\left(2+\frac{\delta}{2}\right) \cos s-\frac{\delta}{2} \cos (\omega s) .
$$

Since $0<\omega<1$, this takes values greater than $2+\frac{\delta}{2}$ for an infinite number of values of $\lambda$. This proves that the curve $y=f(\lambda)$ intersects the straight line $y=2 \cosh k$ an infinite number of times if $|k|$ is not very large.

Remark 2 In the proof of Theorem 3 it appears very clearly that a given problem can be put in the form (3.1)-(3.2) but that the choices of the functions $h(t)$ and $r(t)$ are not unique. The Remark 1 shows that this lack of uniqueness does not generally allow us to take $r \equiv 1$. We want to point out some more about the possible choices of $r$. If $\Psi:[0,1] \rightarrow(0, \infty)$ is a $\mathcal{W}^{2,1}$ function, then the changes

$$
\tau=P^{-1 / 2} \int_{0}^{t} \sqrt{\Psi(s)} d s \text { with } P=\left(\int_{0}^{1} \sqrt{\Psi(t)} d t\right)^{2} ; w(\tau)=\Psi(t)^{1 / 4} u(t),
$$

bring the problem into the form

$$
\begin{gathered}
\frac{d^{2} w}{d \tau^{2}}+\left(\frac{3}{16} \Psi^{-2}\left(\frac{d \Psi}{d \tau}\right)^{2}-\frac{1}{4} \Psi^{-1} \frac{d^{2} \Psi}{d \tau^{2}}+P h \Psi^{-1}\right) w+\lambda P \frac{r}{\Psi} w=0 \\
w(1)=e^{k} w(0) ; \frac{d w}{d \tau}(1)=e^{k} \frac{d w}{d \tau}(0)
\end{gathered}
$$

So, one can replace $r(t)$ by the product of $r$ by an arbitrary positive function of class $\mathcal{W}^{2,1}$. 


\section{$5 \quad$ Algebraic and geometric multiplicities}

Theorem 4 Suppose that $h, r \in L^{1}(0,1)$. The eigenvalues for the problem (3.1)(3.2) (with $k \neq 0$ ) have geometric multiplicities equal to 1 ; if any of them is not real, then its algebraic multiplicity is also equal to 1.

Proof The geometric multiplicity of $\lambda$ is 2 if and only if the matrix of the system (3.3) has rank 0 , but, by using that $\phi(t) \psi^{\prime}(t)-\phi^{\prime}(t) \psi(t)=1$, one deduces that $k=0$. So, all the eigenvalues have multiplicity 1 for $k \neq 0$. Suppose now that $\lambda$ is a non real eigenvalue and $y$ its eigenfunction. For $u=e^{-k t} y$ we have

$$
\begin{aligned}
& u^{\prime \prime}+2 k u^{\prime}+q u+\lambda r u=0, \\
& u(0)=u(1) ; u^{\prime}(0)=u^{\prime}(1) .
\end{aligned}
$$

Consider the operators $L$ and $A$ defined by $L u=\frac{1}{r}\left(u^{\prime \prime}+2 k u^{\prime}+q u\right), A=L+\lambda I$, and $A^{*}=L^{*}+\bar{\lambda} I$. It is known that $L^{*} u=\frac{1}{r}\left(u^{\prime \prime}-2 k u^{\prime}+q u\right)$ with the same domain as $L$ and the metric $(u, v)=\int_{0}^{1} r u \bar{v} d t$.

We have $A u=0$; the algebraic multiplicity of $\lambda$ is greater than 1 if there exists $\tilde{u}$ such that $A \tilde{u}=u$. Let $v$ be such that $A^{*} v=0$. Then, $(A \tilde{u}, v)=(u, v)$ and also $(A \tilde{u}, v)=\left(\tilde{u}, A^{*} v\right)=(\tilde{u}, 0)=0$. Consequently, $(u, v)=0$. But this is impossible by the following

Lemma 2 Suppose that $\lambda$ is not real and $A, A^{*}$ as defined above. If $A u=0$ and $A^{*} v=0$, then $(u, v) \neq 0$.

Proof The function $\bar{v}$ verifies $\bar{v}^{\prime \prime}-2 k \bar{v}^{\prime}+q \bar{v}+\lambda r \bar{v}=0$. Putting $\bar{v}=e^{2 k t} w$, we obtain for $w$ the equation $w^{\prime \prime}+2 k w^{\prime}+q w+\lambda r w=0$.

First let us see that $u(t) \neq 0$ for all $t$. Otherwise, being $u$ a periodic function, we would have $u\left(t_{0}\right)=u\left(1+t_{0}\right)=0$ and $u$ would be an eigenfunction for the Dirichlet problem (3.1) on $\left[t_{0}, 1+t_{0}\right]$ with eigenvalue $\lambda$ and $q(t), r(t)$ extended by periodicity, and $\lambda$ would have to be real. By analogous arguments with Dirichlet, Neumann and third kind conditions we can see that $u^{\prime}(t) / u(t)$ is not real and

$$
v(t) \neq 0, u^{\prime}(t) \neq 0, v^{\prime}(t) \neq 0, v^{\prime}(t) / v(t) \notin \mathbb{R} \text { for all } t .
$$

Also it is clear that $w(t) \neq 0$ for all $t$.

Let us see now that $\frac{w^{\prime}(t)}{w(t)}-\frac{u^{\prime}(t)}{u(t)} \neq 0$ for all $t$. We easily obtain $w^{\prime} / w=-2 k+$ $\bar{v}^{\prime} / \bar{v}$. Using the polar form for $\bar{v}$ and $u$, we can see that $\operatorname{Im}\left(\bar{v}^{\prime} / \bar{v}\right)=\theta \frac{\prime}{v} ; \operatorname{Im}\left(w^{\prime} / w\right)=$ $\theta_{\bar{v}}^{\prime} ; \operatorname{Im}\left(u^{\prime} / u\right)=\theta_{u}^{\prime}$. The substitution of $u=\rho_{u} e^{i \theta_{u}}$ and $\lambda=a+b i$ in (5.1)-(5.2) implies

$$
\theta_{u}^{\prime \prime}+2\left(k+\rho_{u}^{\prime} / \rho_{u}\right) \theta_{u}^{\prime}+b r=0 ; \quad \theta_{u}^{\prime}(1)=\theta_{u}^{\prime}(0)
$$


Solving this equation we obtain

$$
\theta_{u}^{\prime}(t)=\frac{-b e^{-2 k t}}{\rho_{u}^{2}(t)}\left(\frac{1}{e^{2 k}-1} \int_{0}^{1} r \rho_{u}^{2} e^{2 k s} d s+\int_{0}^{t} r \rho_{u}^{2} e^{2 k s} d s\right)
$$

and we deduce that $\theta_{u}^{\prime}(t) \neq 0$ for all $t$ and the sign of $\theta_{u}^{\prime}$ depends on the sign of $k$. Doing the same for $\bar{v}$, the consequence is that $\operatorname{Im}\left(\bar{v}^{\prime} / \bar{v}\right)$ and $\operatorname{Im}\left(u^{\prime} / u\right)$ have different signs, thus $\frac{w^{\prime}(t)}{w(t)}-\frac{u^{\prime}(t)}{u(t)} \neq 0$ for all $t$. Then, we can write

$$
r u \bar{v}=r u w e^{2 k t}=r e^{2 k t} \frac{u w^{\prime}-u^{\prime} w}{w^{\prime} / w-u^{\prime} / u}
$$

Since $u, w$ are solutions of the same differential equation and $u$ is periodic but $w$ is not (remember $k \neq 0$ ), they are linearly independent: $u(t) w^{\prime}(t)-u^{\prime}(t) w(t) \neq 0$ for all $t$, and multiplying $u$ by an appropriate scalar we can assume $u(0) w^{\prime}(0)-$ $u^{\prime}(0) w(0)=1$. Using the Liouville expression for the Wronskian we can finally write

$$
r(t) u(t) \bar{v}(t)=\frac{r(t)}{w(t)^{\prime} / w(t)-u^{\prime}(t) / u(t)} .
$$

This has non-zero imaginary part for all $t$, so

$$
\int_{0}^{1} r(t) u(t) \bar{v}(t) d t \neq 0
$$

Theorem 5 Suppose that $h, r \in L^{1}(0,1)$. The eigenvalues for the problem (3.1)(3.2) have algebraic multiplicities less than or equal to 2.

Proof Because of Theorem 4 above, if $\lambda$ is a multiple eigenvalue it must be real, and, with the same notations as in the proof of that theorem, the equations $(L+\lambda I) u=0$ and $\left(L^{*}+\lambda I\right) v_{a}=0$ do have nontrivial solutions. We suppose also that the algebraic multiplicity is greater than one, so the equation $(L+\lambda I) w=u$ has also a solution. This is equivalent to say that

$$
\int_{0}^{1} r(t) u(t) v_{a}(t) d t=0
$$

We have to prove that the algebraic multiplicity does not exceed two, or, equivalently, that the equation $(L+\lambda I) z=w$ has no solution. This is equivalent to say that

$$
\int_{0}^{1} r(t) w(t) v_{a}(t) d t \neq 0
$$


Let us define $v=e^{-2 k t} v_{a}$. It is clear that $u$ and $v$ satisfy the same differential equation, but are linearly independent, because $u$ and $v_{a}$ are periodic, and so $v$ is not. So, by Liouville's Theorem, there exists $A \neq 0$ such that

$$
u v^{\prime}-u^{\prime} v=A e^{-2 k t} \text {. }
$$

We use the variation of constants method in the equation

$$
w^{\prime \prime}+2 k w^{\prime}+q w+\lambda r w=r u
$$

and look for $w$ in the form $w=C_{1}(t) u(t)+C_{2}(t) v(t)$. By imposing the auxiliary condition $C_{1}^{\prime} u+C_{2}^{\prime} v \equiv 0$, this means that $C_{1}^{\prime} u^{\prime}+C_{2}^{\prime} v^{\prime}=r u$, and these two equations give

$$
\begin{gathered}
C_{1}^{\prime}=-\frac{1}{A e^{-2 k t}} r u v=-\frac{1}{A} r u v_{a}, \\
C_{2}^{\prime}=\frac{1}{A e^{-2 k t}} r u^{2} .
\end{gathered}
$$

Because of condition (5.3) we see that all the functions $C_{1}(t)$ that satisfy $(5.4)$ are automatically periodic. So, imposing the condition of periodicity to $w(t)$ is equivalent to imposing it to the term $C_{2}(t) v(t)$. If we write this term as $C_{3}(t) v_{a}(t)$, with $C_{3}(t)=C_{2}(t) e^{-2 k t}$, we see that $C_{3}(t)$ has to be periodic and that equality (5.5) becomes

$$
C_{3}^{\prime}+2 k C_{3}=\frac{1}{A} r u^{2} .
$$

This is a first order non-homogeneus o.d.e. with constant coefficients, so its general solution is easily obtained. And one easily obtains that its only periodic solution is

$$
C_{3}(t)=\frac{e^{2 k}}{e^{2 k}-1} \frac{1}{A} \int_{0}^{1} e^{-2 k s} r(t-s) u^{2}(t-s) d s,
$$

where the functions $r$ and $u$ are also considered as periodically extended. From this formula we see that $C_{3}(t)$ is of constant sign. Putting all these things together we have

$$
\int_{0}^{1} r w v_{a}=\int_{0}^{1} r\left(C_{1} u+C_{3} v_{a}\right) v_{a}=-\int_{0}^{1} C_{1}(t) C_{1}^{\prime}(t) A d t+\int_{0}^{1} C_{3}(t) r(t) v_{a}^{2}(t) d t \neq 0
$$

since the first integral is zero because $C_{1}(t)$ is periodic and the second is nonzero because $C_{3}(t)$ is of constant sign. And this concludes the proof.

Remark 3 Putting together the results of the previous sections it is clear that there can be eigenvalues of algebraic multiplicity 2 and geometric multiplicity 1 for some values of $k \neq 0$ : this must happen when a pair of real eigenvalues collapse to become nonreal for a value of $k \neq 0$. 


\section{References}

[1] S. Angenent and B. Fiedler, The dynamics of rotating waves in scalar reaction diffusion equations, Trans. Amer. Math. Soc. 3072 (1988), 545-568.

[2] J. von Below, Sturm-Liouville eigenvalue problems on networks, Math. Meth. Applied Sciences 10 (1988), 383-395.

[3] R. Carlson, Eigenvalues for Schrödinger operators on planar domains collapsing onto graphs, University of Colorado. Preprint.

[4] S.N. Chow, K. Lu and J. Mallet-Paret, Floquet bundles for scalar parabolic equations, Arch. Rational Mech. Anal. 129 (1995), 245-304.

[5] E. A. Coddington and N. Levinson, Theory of ordinary differential equations, McGraw-Hill 1955.

[6] J. B. Conway, Functions of one complex variable, Springer-Verlag 1978.

[7] E. Giusti, Minimal surfaces and functions of bounded variation, Birkhäuser 1984 .

[8] J. K. Hale and G. Raugel, A reaction-diffusion equation on a thin L-shaped domain, Proc. Royal Soc. Edinburgh, Sec. A 125 (1995), 283-327.

[9] T. Kato, Perturbation theory for linear operators, Springer Verlag 1980.

[10] B. M. Levitan and I. S. Sargsjan, Sturm-Liouville and Dirac operators, Kluwer Academic 1991.

[11] J. A. Lubary, Multiplicity of solutions of second-order linear differential equations on networks, Linear Algebra and its Applications 274 (1998), 301-315.

[12] J. A. Lubary, Doctoral Thesis, Universitat Politècnica de Catalunya. In preparation. 